\title{
A Computational Learning Approach for the Development of Karate Sequences
}

\author{
David Newth ${ }^{*}$, Stuart McDonald ${ }^{2}$ \\ ${ }^{1}$ Australian Okinawan Goju-ryu Karate-do Federation, Woden Dojo, Canberra, Australia \\ ${ }^{2}$ Kinetic Medicine, Albury, Australia \\ Email: *david.newth@gmail.com
}

How to cite this paper: Newth, D., \& McDonald, S. (2021). A Computational Learning Approach for the Development of Karate Sequences. Advances in Physical Education, 11, 503-512. https://doi.org/10.4236/ape.2021.114041

Received: September 28, 2021 Accepted: November 27, 2021 Published: November 30, 2021

Copyright () 2021 by author(s) and Scientific Research Publishing Inc. This work is licensed under the Creative Commons Attribution International License (CC BY 4.0).

http://creativecommons.org/licenses/by/4.0/

Open Access

\begin{abstract}
Karate, like most martial arts, relies on the development of complex sequences of interrelated techniques. The development of strong technique, physical endurance, mobility, and precision requires repetitive practice of sequences and combinations of moves. However, training typically has biases, leading to limited repertoire, and poor dynamical decision making. Here we use machine learning to develop a mathematical model of sequences of karate techniques. We present a series of algorithms for the generation of novel training combinations, which are internally consistent with a supplied training regime. Due to the general nature of the mathematical approach developed here, we anticipate our approach has wider applications, for example analysing individual competitors' decision-making process and performance, identifying weaknesses and vulnerabilities in an athlete's repertoire.
\end{abstract}

\section{Keywords}

Martial Arts, Machine Learning, Sequence Training, Kata, Kumite

\section{Introduction}

The World Karate Federation (WKF) estimates that there are over 10 million athletes and 100 million karate practitioners worldwide (Stefano Vando et al., 2013), making karate one of the most popular martial art/combat sports (Koropanovski et al., 2011). At a competition level, Karate is divided into two different specialities: Kumite and Kata. Kumite (sparing) is non-contact combat between two opponents using punching, kicking, and striking techniques. Kata (forms) represents real combat with imaginary opponents. Both disciplines require an athlete to perform complex high momentum and velocity techniques with precision, requiring physical conditioning (particularly anaerobic capacity) 
(Beneke et al., 2004), postural control (Filingeri et al., 2012), tactical and technical skill (Cesari \& Bertucco, 2008). Hironori Ōtsuka (2006), the founder of Wado-Ryu karate, emphasised that the athlete must be prepared to move or execute a strike in any direction at any moment in time. This requirement is reflected in both kumite and kata training.

Kumite engages an opponent, within a controlled combat environment. The dynamic nature of kumite ensures the athlete must be agile (changing direction in response to stimuli) and possess a high anaerobic capacity (Beneke et al., 2004). As the athlete engages their opponent, they will be required to both defend themselves against attacks and evade their opponent's defences in an effort to reach their target, in order to score points. Analysis of 1198 kumite sequences during the 1992 European championships found that $11.4 \%$ of combinations lasted between 1 - 7 seconds, while 79.5\% lasted between 8 - 50 seconds (Sterkowicz \& Franchini, 2009). The athlete must have a high degree of agility and control to string together effective combinations.

Kata, by contrast, are sequences of techniques designed to teach the proponent real-world sequences that apply to a self-defence situation. As a discipline, Kata requires the athlete to move their body with precision in multiple directions within a sequence, during which the body may be required to move left, right, forward, backward, rotate 180 degrees or more. Blocks, kicks and strikes follow each other, and there may be requirements for jumping and for rotating the body around an axis while jumping through the air (Camomilla et al., 2009).

Progression in both disciplines requires the development of, and familiarisation with, sequences of moves, in order to gain physical control of moving into, out of, and between techniques. The process of learning these techniques and conditioning the body to perform them efficiently, involves learning smaller pre-choreographed sequences of kicks, strikes and postures/stances; often taught in a training class as Kihon Ido (moving fundamentals) or Renraku Waza (follow-up techniques). From here, ability is developed by growing the athlete's skill in both more advanced techniques and more complex combinations of techniques. These combinations teach the body how to redirect its kinetic energy effectively, while maintaining postural control (Filingeri et al., 2012).

The sequences of techniques within a kata or kumite sequence are far from random. For example, analysis of kumite shows that mawashi-geri (roundhouse kick) and gyaku-zuki (reverse punch) are the most effective techniques, and therefore effective representative combinations need to be constructed around them to maximise their effectiveness. Similarly, kata is broken down into sequences to assist in learning and development of postural strength and control, and technical correctness; again, the development of supplementary training sequences is important for physical conditioning and overall agility.

The development of effective technique training is central to athlete development in karate. In this paper, we propose the use of a computational learning approach to assist in the development of Renraku Waza sequences to support 
kumite and kata training. The algorithm is presented with sequences (that can be taken from a kata, grading syllabus or observed kumite sequences), learns the statistical relationships between moves, and generates new combinations based on those relationships. The use of computational and machine learning techniques, for the development of Renraku Waza techniques, has at least four advantages:

By learning the statistical relationships between moves, and preserving those relationships in the generated sequences, the created combinations are internally consistent, yet novel, with the syllabus presented to the algorithm.

The agnostic nature of the algorithm means there is no human bias towards preferred techniques or avoidance of difficult techniques. It is well recognized that when presented with a decision around actions, the psychology of humans will favour those (in this case) techniques that are familiar and present less risk. The result is a more rounded training syllabus.

Aiding the coaches to understand the importance of certain sequences for learning purposes and to formulate an objective, stepwise approach to building motor skills in athletes.

Coaching the coaches, giving them a bigger set of sequences to choose between. Provides a consistent tool for the development of sequences and progressions.

As an example, we will apply our approach to the development of training sequences that will support Wado-Ryu grading syllabus. The remainder of this paper is organized as follows. In the following section, we outline Renraku Wa$\mathrm{za}$, the data and mathematical approach to representing the relationship between moves, and finally, we present 3 algorithms for generating combinations of moves with various properties. In section 3, we provide some examples of the Renraku Waza generated by our approach. In section 4 , we provide a discussion of the implications, applications and next steps in this research. Finally, in section 5, we provide some closing remarks.

\section{Methods}

During a kata or kumite bout, there are three possible situations an athlete could face: attack (kick, punch, advance...), defend (block, counter, tai sabaki...), and withdraw (turn, evade, retreat). Each of these situations, be it kata or kumite, change during a bout. The selection of techniques, timing, and postural control requires practice and experimentation and is taught within the context of a training syllabus for a particular style of karate. In this section, we develop a formal mathematical model of a syllabus. We then take the Wado-Ryu grading syllabus and encode it within the framework outlined here.

\subsection{A Mathematical Model of Technique Sequences}

We define a model of a collection of techniques, or syllabus, as a simplified finite state machine (Hopcroft \& Ullman, 1979), that has a set of moves and a set of transitions between each move. Formally, the syllabus, $\mathcal{M}$ is a four-element tuple: 


$$
\mathcal{M}=S, S_{I}, S_{F}, \delta \text {, }
$$

where, $S$ is a finite set of moves $\left\{s_{1}, s_{2}, \cdots, s_{n}\right\}$, that make up the entire syllabus. $S_{I}$ is a finite set of starting or initial sequence moves, and $S_{I} \subseteq S$. $S_{F}$, is a finite set of ending or terminal moves in the sequence, with $S_{F} \subseteq S$. It should be noted that for the syllabus to be complete, the conditions: $S_{I} \neq \phi$ and $S_{F} \neq \phi$ must hold. $\delta$, is a set of production functions of the form $\delta: s_{i} \otimes \operatorname{Pr}\left(s_{j}\right) \mapsto s_{j}$, which can be read as technique $s_{j}$ follows from technique $s_{i}$ with some probability $\operatorname{Pr}\left(s_{j}\right)$.

As Green (1990) demonstrates, the set of production functions $\delta$ can be rewritten as a directed graph $G$. In formal terms, a digraph is an ordered pair $G=(S, A)$, where $S$ is the set of moves within the syllabus and referred to as vertices or nodes, and $A$ is a set of ordered pairs of vertices $\left(s_{i}, s_{j}\right)$, where $A \subseteq\left\{\left(s_{i}, s_{j}\right) \mid s_{i}, s_{j} \in S^{2}\right\}$, that depicts an arc from $s_{i}$ to $s_{p}$ or that $s_{j}$ follow on from $s_{i}$. Each arc is assigned a weight $a_{i} \in[0,1]$, that represents the probability that technique $s_{j}$ follow from $s_{i}$. Figure 1 illustrates how a sequence of moves is encoded into a graph, so the final model of a syllabus can be written as:

$$
\mathcal{M}=\left\langle S, S_{I}, S_{F}, G(S, A)\right\rangle \text {. }
$$

\subsection{Data}

Many styles of karate exist, but only four are recognised by the World Karate Federation (WKF): Goju, Shito, Shotokan and Wado. Each of style follows specific philosophies and principles, which results in variations of techniques (WKF, 2020). For the purposes of illustration we have chosen to apply the approach here to Wado Ryu grading syllabus (MacClean, 1990); this choice is arbitrary and can be applied to any style of karate. Wado-Ryu is a recognized

Technique

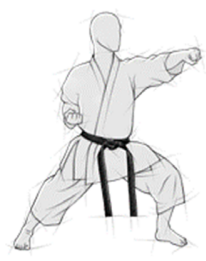

Front Punch

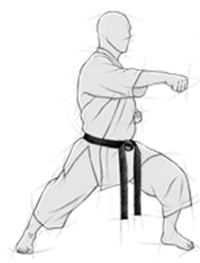

Reverse Punch

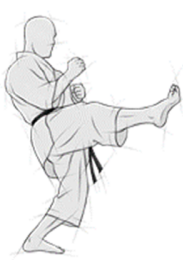

Front Kick

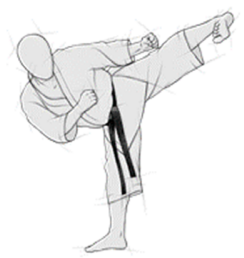

Roundhouse Kick

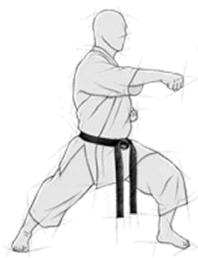

Reverse Punch
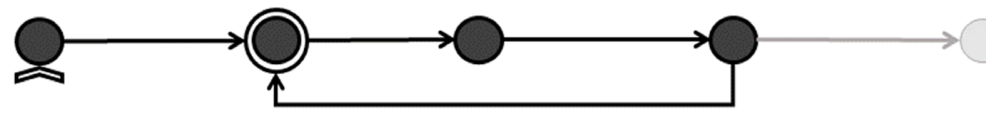

Graph Representation

Figure 1. Encoding of techniques as a graph. Each technique is represented as a node (circle). All the unique techniques for the set $S$. The relationship between techniques (e.g. reverse punch follows a front punch), are depicted by arrows (arcs), and the sets $S$ and $A$ make up the graph, $G(S, A)$. The chevron associated with front punch node, indicates that front punch is an initial or starting technique (i.e. front punch is a member of the set $S_{I}$. Finally the double circle on reverse punch indicates that it is a final technique and is a member of the set $S_{F}$. 
independent style of karate founded in 1934 by Master Hironori Ötsuka and is derived from a combination of Shotokan Karate and Ju-jitsu (Wado-Ryu Karate-Do Renmei, 2018). The Wado-Ryu style has a number of unique characteristics, including its emphasis on not just striking but tai-sabaki (body evasion). Another characteristic is its applied nature of the style, with the style taught as a progression from kihon to kata to kumite. Due to the applied nature of WadoRyu, each grade is required to display a set of competencies in Renraku Waza. These competencies are clearly codified in the Wado-Ryu grading syllabus (MacClean, 1990). Therefore, the development, progressions and variations are an important part of the development of the athlete's ability. As the test data for the approach here, we have used the Wado Ryu Australia grading syllabus for $10^{\text {th }}$ Kyu to $1^{\text {st }}$ Dan (MacClean, 1990). It should be noted, that while we have applied the techniques here to the Renraku Waza component of the syllabus, it can be easily applied to kihon, kata or kumite, as long as there are enough input data, and that data can be mapped to satisfy Equation 2 above.

\subsection{Encoding Data}

Every technique within the training syllabus is assigned to the set $S$. For the Wado-Ryu grading syllabus, we identified 25 distinct techniques. Overall, the syllabus contained 8 punching techniques, four blocking techniques, 11 kicking techniques, and two additional techniques: a turn (Mawatte) and a step (Surikomi). We treated punches and kicks to different regions of the body (e.g. Sokuto Chudan and Sokuto Jodan) as separate techniques, as these techniques require different levels of postural control. Techniques have previously been classified according to the target location, strike direction, shape of the line of attack and the body part performing the attack (Mudric \& Rankovic, 2016). When punching to the head or body, for example, the location of the centre of mass changes relative to the limb length and height of the target, which changes the direction of centre of mass.

Techniques that started a sequence were added to the set $S_{F}$. While techniques that ended a sequence were added to the set $S_{F}$. The graph $G$, was represented as an adjacency matrix M. Each combination of techniques within the syllabus was parsed, and the frequency of occurrence of each pair of techniques $\left(s_{i}, s_{j}\right)$ was recorded in $A_{i r}$. Once all pairs of techniques were parsed, the frequencies of occurrence were normalised into a probability of $s_{j}$ following $s_{i}: W_{i j}=\frac{A_{i j}}{\sum_{k} A_{i k}}$. The resulting weighted graphy is shown below in Figure 2 .

\section{Generated Sequences}

The model described in Equation (2) contains the statistical, semantic and syntactic information about the relationships, between moves within a syllabus. By performing various walks upon the graph $G$, that are consistent with model $\mathcal{M}$, we can create sequences of techniques that preserve statistical, semantic and syntac- 
The inclusion of backtracking, and other constraints, such as a prescribed walk length, allows the development of sequences of combinations that of a defined length. Algorithm 2 shows the refinements to the initial algorithm, and Figure 4 provides examples of sequences of different prescribed lengths.

From the algorithm presented above, further refinements, the inclusion of backtracking, allows for the development of progressions, where a set of moves is executed, and the last step is removed and replaced with a new sub sequence of moves. Such training allows for the athlete to develop dynamic decision-making capability and to develop familiarity between alternative courses of action. Figure 5 below, illustrates the output of such an algorithm, where the sequences are built up from 3 techniques to 16 .

\section{Discussion}

This paper has been focused on the development of a mathematical model and its application to the development of training sequences. The approach here has several wider applications in the applied domain. As outlined in section 3, the mathematical model detailed in section Equation (1), can be expanded to include opponent counter attacks. This leads to an interesting application in kumite. If sequences of exchanges (combinations of attack, and counter attacks),

Algorithm 2. A simple algorithm for creating sequences of length L.

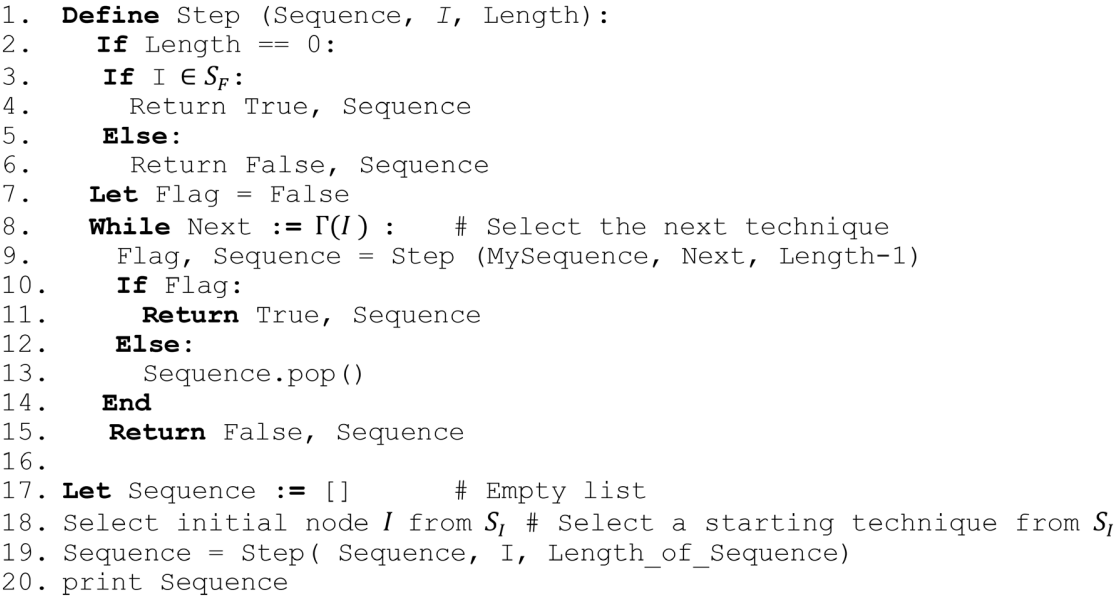

Figure 4. Three generated sequences of a prescribed length. The sequences much conform to the start and end conditions outlined in Equation (2). 


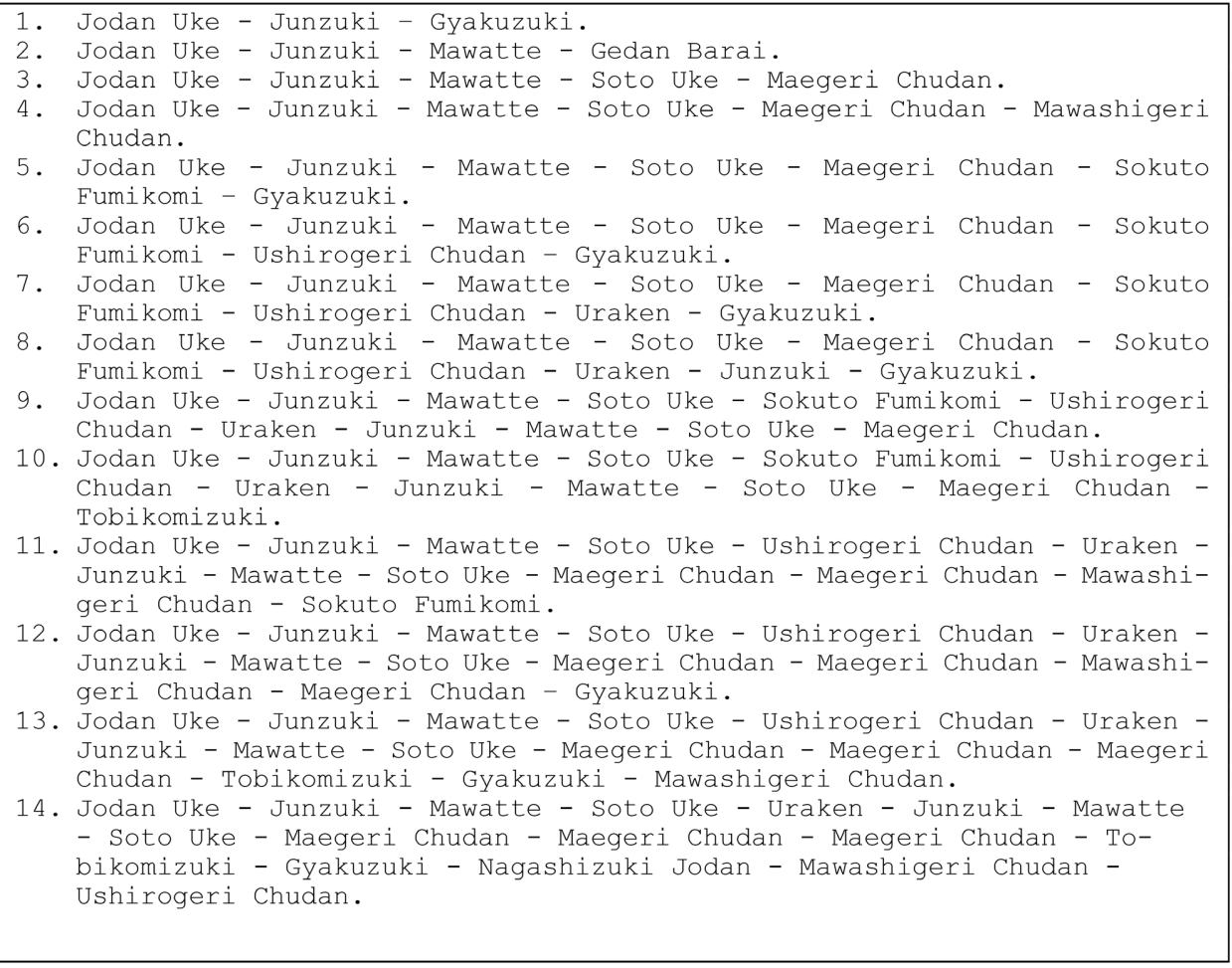

Figure 5. Illustration of the output of the recursive algorithm, with backtracking to develop longer and longer training sequences. The sequences must conform to the conditions outlined in Equation (1).

from (say) international matches, as per (WKF, 2020), it would become possible to develop a model of kumite, and therefore develop training sequences that are more likely to lead to scoring opportunities, and away from sequences that lead to less favourable outcomes. Given enough observations of an individual, it would be possible to build a model of an athlete's kumite repertoire, techniques and counters; in effect, the mathematical model is capturing and reproducing an athlete's mental model and decision-making skills. Analysis of this model could be used to reveal strengths and weaknesses. Comparison between an athlete and the wider set of successful athletes could highlight areas for development. Likewise, knowledge of an athlete's mental model and sequence repertoire, could be used in training to give opponents a strategic advantage in a match.

From a cognitive developmental perspective, analysis of models of different skill levels, e.g. beginner to international competitor, would provide useful insight. The application of algorithmic information theory (Kolmogorov, 1963; Chaitin, 1966), would provide insights into the cognitive development of athletes, as well as the complexity of decision making of different skill levels and different athletes.

The approach is equally valid for kata training, where the sequences of particular katas can be codified and training sequences developed. The purpose of this would be to improve transitional strength between kata positions (postures) and cognitive function. Kata is often considered a training program in and of itself. 
However, the body must have the conditioning to perform the sequences of movements that kata demands. By codifying and sequencing movement combinations according to the need of kata, these sequences can be taught to the athlete, who can then work on their specific areas of need. This has the advantages of improving their physical capability generally (improving their body's ability to perform the movements in sequence, which has transference to kumite) and of improving their kata specifically. Rather than focusing on the entire kata sequence, they can work on their body's movement efficiency outside of kata (but with movements that correspond to kumite), in a systematic, logical and applied manner.

Within the context of Kihon, Kata and Renraku Waza, where we are simply interested in developing sequences of techniques, the production functions outlined in section 2.2 are adequate. However, in the context of Kumite, where there is an active opponent, an additional set can be added to $\mathcal{M}$, so

$\mathcal{M}=S, \Sigma, S_{I}, S_{F}, \delta$, where $\Sigma$ is the set of opponent moves, and the production functions become $\delta: s_{i} \otimes \varepsilon_{k} \mapsto s_{j}$, which reads, technique $s_{j}$ follows if, $s_{i}$ has been completed and the opponent counters with $\varepsilon_{k}$. Again, the analysis of underlying relationships would provide useful insights into training development as well as the performance of athletes. For example, given examples of kumite bouts between rivals, common attacks and counterattack sequences could be identified and counter or alternative move sequences that maximise an athlete's success determined.

\section{Acknowledgements}

The authors would like to thank Fiore Tartaglia for providing the template illustrations upon which Figure 1 was developed.

\section{Conflicts of Interest}

The authors declare no conflicts of interest regarding the publication of this paper.

\section{References}

Beneke, R., Jachner, C., Erasmus, J., \& Hütler, M. (2004). Energetics of Karate Kumite. European Journal of Applied Physiology, 92, 518-523.

https://doi.org/10.1007/s00421-004-1073-x

Camomilla, V., Sbriccoli, P., Di Mario, A., Arpante, A., \& Felici, F. (2009). Comparison of Two Variants of a Kata Technique (Unsu): The Neuromechanical Point of View. Journal of Sports Science and Medicine, 8, 29-35.

Cesari, P., \& Bertucco, M. (2008). Coupling between Punch Efficacy and Body Stability for Elite Karate. Journal of Science and Medicine in Sport, 11, 353-356. https://doi.org/10.1016/j.jsams.2007.05.007

Chaitin, G. J. (1966). On the Length of Programs for Computing Finite Binary Sequences. Journal of the ACM, 13, 547-569. https://doi.org/10.1145/321356.321363

Filingeri, D., Bianco, A., Zangla, D., Paoli, A., \& Palma, A. (2012). Is Karate Effective in 
Improving Postural Control? Archives of Budo, 8, 203-306.

Green, D. G. (1990). Syntactic Modeling and Simulation. Simulation, 54, 281-286. https://doi.org/10.1177/003754979005400605

Hopcroft, J., \& Ullman, J. (1979). Introduction to Automata Theory, Languages, and Computation. Addison-Wesley.

Knuth, D. (1997). The Art of Computer Programming. 1: Fundamental Algorithms (3rd ed.). Addison-Wesley Professional.

Kolmogorov, A. (1963). On Tables of Random Numbers. Sankhyā Series A, 25, 369-375.

Koropanovski, N., Berjan, B., Bozic, P. R., Pazin, N., Sanader, A., Jovanovic, S., \& Jaric, S. (2011) Anthropometric and Physical Performance Profiles of Elite Karate Kumite and Kata Competitors. Journal of Human Kinetics, 30, 107-114. https://doi.org/10.2478/v10078-011-0078-x

MacClean, W. (1990). Wado Ryu Australia: Official Grading Book. Wodo Ryu of Japan Australia.

Mudric, R., \& Rankovic, V. (2016). Analysis of Hand Techniques in Karate. SPORT-Science \& Practice, 6, 47-74.

Ōtsuka, H. (2006). Wado Ryu KarateWado Ryu Karate: Hironori Ōtsuka. Rising Sun Productions, Japan.

Stefano Vando, S., Filingeri, D., Maurino, L., Chaabène, H., Bianco, A., Salernitano, G., Foti, C., \& Padulo, J. (2013). Postural Adaptations in Preadolescent Karate Athletes Due to a One Week Karate Training Camp. Journal of Human Kinetics, 38, 45-52. https://doi.org/10.2478/hukin-2013-0044

Sterkowicz, S., \& Franchini, E. (2009). Testing Motor Fitness in Karate. Archives of Budo, 5, 29-34.

Wado-Ryu Karate-Do Renme (2018). History. http://www.wado-ryu.jp/en/data/history.pdf

World Karate Federation (WKF) (2020). Karate Competition Rules. WKF Kata and Kumite Competition Rules for 2020. https://www.wkf.net/structure-statutes-rules 